Revue des patrimoines

$21 \mid 2013$

De l'art de bâtir aux champs à la ferme moderne

\title{
Le Mouvement moderne aux champs. De la Ferme radieuse au Regional Planning
}

\section{Guillemette Morel Journel}

\section{(2) OpenEdition}

\section{Journals}

Édition électronique

URL : http://journals.openedition.org/insitu/10458

DOI : 10.4000/insitu. 10458

ISSN : 1630-7305

\section{Éditeur}

Ministère de la culture

Référence électronique

Guillemette Morel Journel, « Le Mouvement moderne aux champs. De la Ferme radieuse au Regional Planning », In Situ [En ligne], 21 | 2013, mis en ligne le 17 juillet 2013, consulté le 03 mai 2019. URL : http://journals.openedition.org/insitu/10458; DOI : 10.4000/insitu. 10458

Ce document a été généré automatiquement le 3 mai 2019.

\section{(c) $($ i) $(9)$}

In Situ Revues des patrimoines est mis à disposition selon les termes de la licence Creative Commons Attribution - Pas d'Utilisation Commerciale - Pas de Modification 4.0 International. 


\title{
Le Mouvement moderne aux champs. De la Ferme radieuse au Regional Planning
}

\author{
Guillemette Morel Journel
}

1 L'expression « ferme moderne ${ }^{1}$ » semble relever de l'oxymore pour l'architecture du $\mathrm{XX}^{\mathrm{e}}$ siècle, tant celle-ci est marquée du sceau du « Mouvement moderne »- quelles que soient les précautions historiographiques que cette notion appelle ${ }^{2}$. En effet, pour les théoriciens comme les praticiens dudit mouvement, l'enjeu est avant tout la réalisation de la Grande Ville. L'aménagement du territoire en général, et agricole en particulier, ne fait pas partie de leurs préoccupations avant la Seconde Guerre mondiale. Il est vrai que le monde nouveau, celui de la machine et de l'homme moderne, trouve alors plus sa place dans les métropoles grandissantes que dans les campagnes, supposées arriérées et sourdes aux appels de la nouveauté - une méconnaissance que l'origine urbaine de la majorité des architectes explique en partie. La réalité est autrement plus subtile. Durant la première moitié du $\mathrm{XX}^{\mathrm{e}}$ siècle, l'agriculture occidentale n'a pas ignoré les révolutions techniques. Mais dans le monde savant des idées architecturales et urbaines, elle ne fait pas l'objet de réflexions et de propositions concrètes. C'est pourquoi on évoquera ici non pas des réalisations matérielles mais des propositions théoriques, non localisées, à une grande échelle - celle de la région.

Dans un terrain laissé en friche par les historiens de l'architecture du $\mathrm{XX}^{\mathrm{e}}$ siècle, prenons pour repères les contributions de deux concepteurs fameux, Le Corbusier (1887-1965) et Ludwig Hilberseimer (1885-1967). Contemporains, le Franco-Suisse et le GermanoAméricain appartiennent à la même famille intellectuelle et artistique, celle de la modernité, du Bauhaus et des Congrès internationaux d'architecture moderne (CIAM). En témoigne leur participation à la cité-exposition du Weissenhof, orchestrée par Ludwig Mies van der Rohe à Stuttgart en 1927. Tous deux ont en effet théorisé et publié sur l'aménagement des espaces ruraux, tous deux en évoquant et nommant explicitement cet objet a priori non architectural qu'est «la ferme». L'examen de leurs propositions, 
conçues dans les années 1930 et 1940, permet d'aborder une question délicate : qu'est-ce que les architectes dudit Mouvement moderne ont pensé, et plus concrètement projeté, sur les territoires non urbains? La rareté de tels projets n'est-elle pas l'indice d'une méconnaissance, voire d'une indifférence, à tout ce qui ne relevait pas de l'espace métropolitain?

3 Avant de présenter les propos de Hilberseimer, évoquons ceux de Le Corbusier sur « La ferme radieuse ». Sans nous attarder sur les sources, le contexte d'élaboration, le dispositif spatial et l'influence du «Village radieux ${ }^{3}$ » de Le Corbusier, mentionnons les différentes occurrences de publication de ce principe qui connut plusieurs versions.

\section{La Ferme radieuse, une réflexion de longue durée}

Le projet de Ferme radieuse - le qualificatif témoigne de la filiation avec la Ville radieuse, développée par Le Corbusier dès le début des années 1930 -, a paru pour la première fois sous le titre «La ferme radieuse, le village radieux, réorganisation agraire " $\left(\right.$ fig. $\left.\mathbf{n}^{\circ} \mathbf{1}\right)$ dans la revue Prélude en novembre $1934^{4}$, et fut repris l'année suivante dans le fort volume La Ville radieuse. Loin de n'être qu'une réponse circonstancielle à l'incitation de l'ouvrier agricole sarthois Norbert Bézard, la thèse de l'article (qui ne compte pas moins de 16 pages) sera répétée à de nombreuses reprises jusqu'à la fin des années 1950. L'originalité de la démarche de Le Corbusier - outre son intérêt pour l'aménagement des campagnes, guère partagé par ses contemporains - réside dans la multiplicité des échelles qu'elle convoque: depuis les détails architecturaux des bâtiments agricoles jusqu'au remembrement parcellaire et au raccordement au réseau national de circulation. La déclinaison rurale de la Ville radieuse s'inscrit donc dans une réflexion globale sur l'aménagement du territoire et forme un concept rigoureusement articulé. 


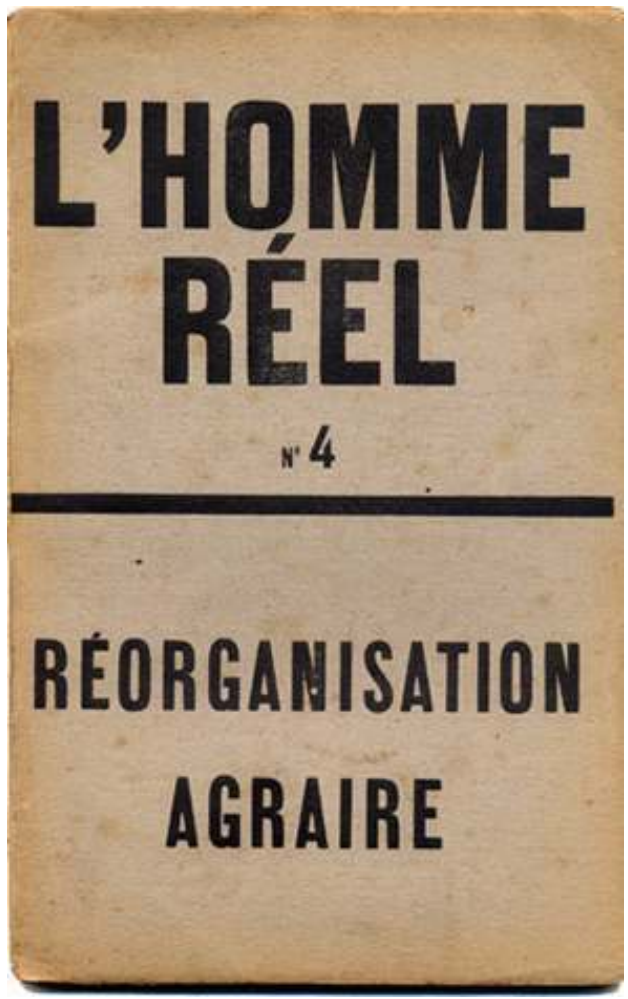

Couverture de la revue L'Homme réel, novembre 1934.

Repro. Morel Journel, Guillemette. (c) Guillemette Morel Journel.

Ainsi, fin 1934, soit en même temps que la publication de l'article de Prélude, dans le deuxième volume de son Euvre complète, Le Corbusier évoque les liens indissolubles qui lient les espaces urbains et ruraux : «Il n'est pas possible de songer à urbaniser les villes modernes si l'on ne pense pas à aménager les campagnes. Une partie des habitants de la ville devra retourner à la campagne. Mais, si la campagne demeure ce qu'elle est aujourd'hui, personne ne voudra y retourner. Il s'agit donc d'opérer le regroupement du sol, de constituer les villages coopératifs et de fixer un nouveau type de ferme, faisant état de l'outillage moderne de culture ${ }^{5}$.»

Dans l'ouvrage accompagnant la construction du Pavillon des temps nouveaux lors de l'Exposition universelle de 1937 (fig. $\mathbf{n}^{\circ} \mathbf{2}$ ), l'architecte consacre plusieurs pages à la Ferme radieuse et au village dont elle fait partie; ce dernier est désormais nommé "Village coopératif ${ }^{6}$ ", afin d'insister sur son mode de gestion. Le Corbusier concède que, depuis vingt années, il ne s'était intéressé qu'aux villes. Il place le problème à l'échelle de la région (perspective élargie qu'il conservera par la suite), laquelle ne vit que par la convergence de « ressources agricoles » et de « ressources industrielles ». S'il propose des solutions, il ne cache pas la gravité de la situation : «Les campagnes meurent, vétustes, fermes et villages écroulés. Fermes et villages à vendre. C'est sur ce soubassement de déchéance que furent peintes les images de la réformation agraire ${ }^{7}$. 

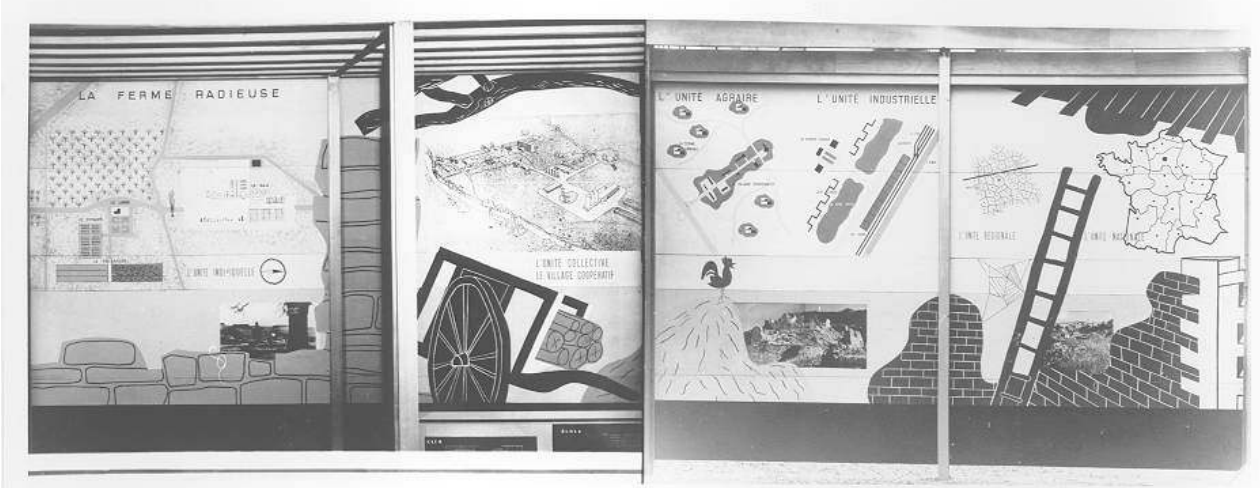

Panneaux muraux présentant les propositions de Le Corbusier pour le monde rural dans le Pavillon des temps nouveaux, Paris, 1937. Fondation Le Corbusier, L2-13-148.

(c) ADAGP/FLC.

7 En 1939, alors qu'il rédige le manuscrit de Sur les 4 routes $^{8}$, livre qui réserve une bonne place au sort des paysans, Le Corbusier publie dans la revue Sillons un long texte intitulé «Renaissance de la vie paysanne». Sa fascination - toute vichyssoise - pour la Terre et ses travailleurs y est opposée avec lyrisme aux risques de la révolution industrielle : « Au travers de cette entité famille-terre, des courants animateurs ou destructeurs ont passé : le souffle de la civilisation machiniste - souffle d'époque, "air du temps", événement ou puissance aussi irrésistible que le jeu des sèves à l'heure d'un nouveau printemps ${ }^{9}$. »

L'année suivante, Le Corbusier s'apprête à publier, aux éditions Fernand Sorlot (qui sortiront son Destin de Paris en 1941), un petit ouvrage intitulé La Ferme radieuse et le Centre coopératif; le livre, prévu dans la collection «Cahiers de politique nationale. Série Prélude ", restera toutefois dans les cartons, alors que la mise en pages et la couverture étaient déjà réalisées (fig. $\mathbf{n}^{\circ} \mathbf{3}$ ). Le texte ${ }^{10}$ reprenait, sur un ton moins sentencieux et souvent autobiographique, les idées qui avaient été présentées dans La Ville radieuse. 


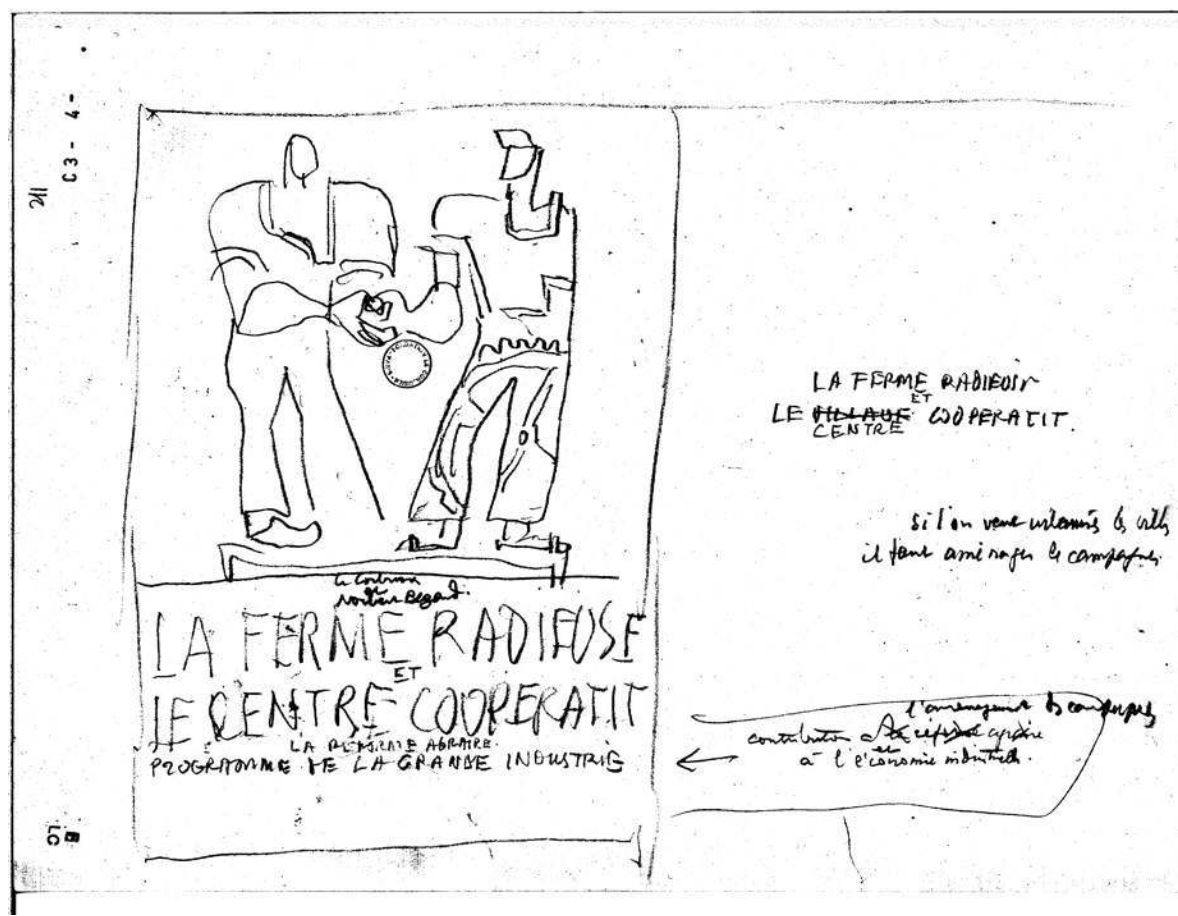

Le Corbusier, projet de couverture pour le livre La Ferme radieuse et le Centre coopératif, 1940. Fondation Le Corbusier, C3-4-212.

(c) ADAGP/FLC.

L'édition originale ${ }^{11}$ de Les Trois Établissements humains, signée du collectif Ascoral mais rédigée presque intégralement par Le Corbusier, dédie en 1945 près de la moitié de ses pages (soit une centaine) à «l'Unité d'exploitation agricole». Celle-ci constitue le pendant de l'Unité d'habitation de grandeur conforme, principe dont il construira dès 1948 un premier exemplaire à Marseille.

Les deux titres parus l'année suivante - Propos d'urbanisme, et Manière de penser l'urbanisme ${ }^{12}$ - ne consacrent en revanche que quelques pages à la question agricole, évoquant ce qui est alors nommé « l'unité rurale ».

Plus de dix ans après, en 1959, la réédition, très remaniée par l'éditeur Jean Petit, des Trois Établissements humains, sous le titre L'Urbanisme des trois établissements humains ${ }^{13}$, revient néanmoins, vingt pages durant, sur « l'Unité d'exploitation agricole » (fig. $\left.\mathbf{n}^{\circ} \mathbf{4}\right)$, héritée de l'ouvrage de l'Ascoral. Elle est présentée comme le premier des trois modes d'occupation de l'espace national: la question rurale reste donc au rang des préoccupations de l'architecte. 


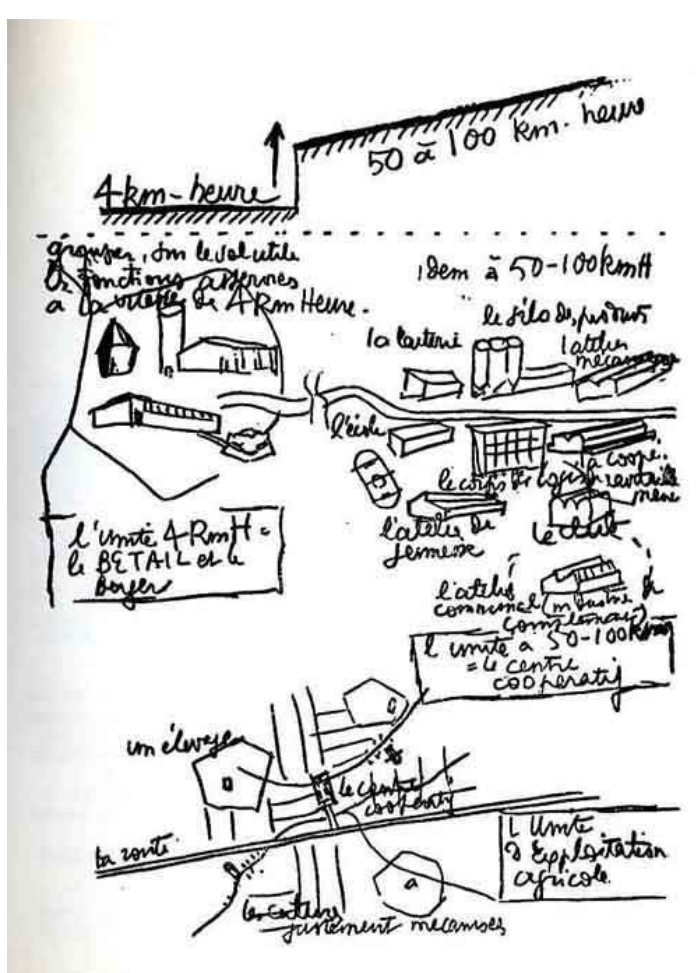

La version de 1959 des Trois Établissements humains présente (p. 79) le principe de l'Unité d'exploitation agricole dans ses rapports avec les différentes vitesses permises par les voies de circulation.

Repro. Morel Journel, Guillemette. (c) Guillemette Morel Journel.

\section{Par-delà la ville et la campagne, la planification régionale}

Considérons, parallèlement aux propositions de Le Corbusier, un ouvrage peu connu en France $^{14}$ de Ludwig Hilberseimer, compagnon de route de Mies van der Rohe à Dessau, Berlin et Chicago ${ }^{15}$ : The New Regional Pattern. Industries and Gardens, Workshops and Farms, publié en 1949 à Chicago (fig. $\mathbf{n}^{\circ}$ 5). Le fait (rare) que le terme de "ferme » soit employé dans le titre, aux côtés de ceux, alors plus courants, de "jardin», "atelier» et « industrie » est remarquable en soi. À ma connaissance, aucune littérature issue du Mouvement moderne n'affiche un quelconque intérêt pour le sujet. Toutefois, l'ouvrage s'avère en cela décevant, puisque les fermes n'y sont analysées que de manière sommaire et contingente, mais la mention explicite de ce thème rend le livre digne d'attention. 
Figure 5

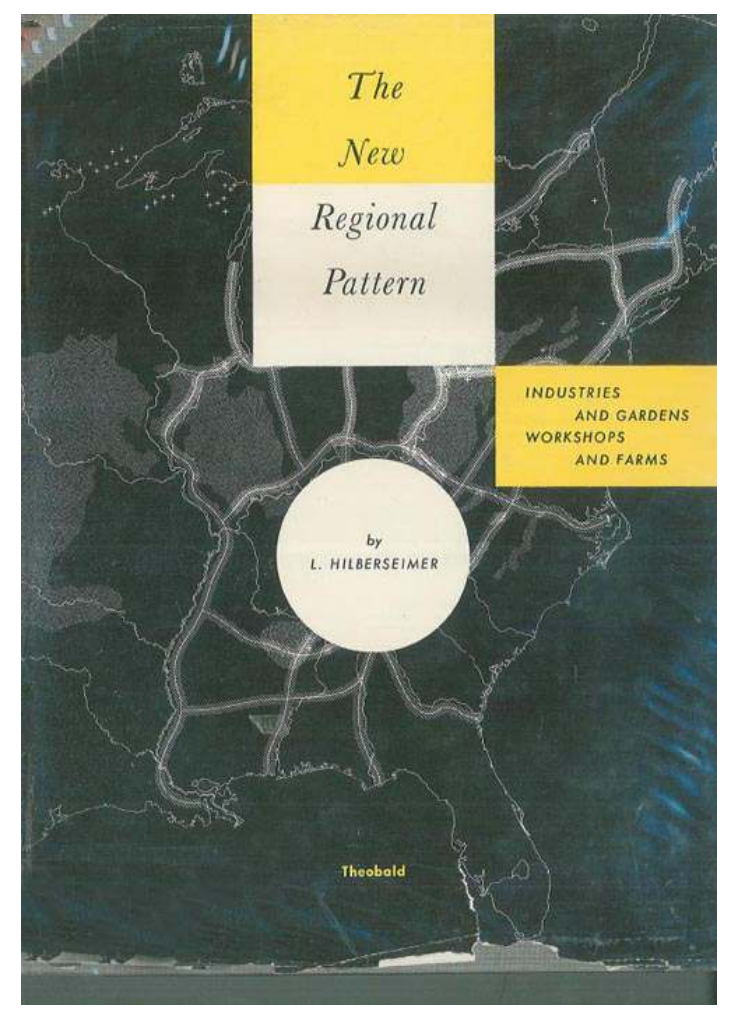

Couverture du livre de Ludwig Hilberseimer sur le Regional Planning, 1949. Repro ??

Repro. Morel Journel, Guillemette. (c) Guillemette Morel Journel.

L'histoire de l'architecture se souvient de Hilberseimer moins en tant qu'architecte qu'enseignant de l'urbanisme, que ce soit au Bauhaus de Dessau ou à l'Illinois Institute of Technology (IIT) à Chicago. Il a publié quelques ouvrages mémorables, parmi lesquels, en 1927, Großstadtarchitektur, et, un an plus tard, Beton als Gestalter; puis, après son émigration aux États-Unis, The New City. Principles of Planning (1944), The Nature of Cities. Origin, Growth, and Decline. Pattern and Form. Planning Problems (1955), suivi d'une monographie de Mies van der Rohe (1956) et de Contemporary Architecture, It's Roots and Trends (1964) ${ }^{16}$. Un an plus tôt, il avait publié dans sa langue d'origine une sorte d'autobiographie intellectuelle retraçant cinquante années de projets pour la ville ${ }^{17}$. Tout au long de sa vie, Hilberseimer alternera ainsi la publication de ses propres théories et la présentation critique des œuvres de ses contemporains.

The New Regional Pattern s'insère dans une véritable série de publications sur l'aménagement et l'architecture. Publié après la guerre, en 1949, il synthétise, ainsi que l'explique l'auteur, une réflexion liée à son enseignement depuis près de vingt ans : «J'ai commencé les recherches pour Le Nouveau Modèle régional au début des années 1930, quand je me suis soudain trouvé avec le loisir nécessaire pour aborder ces problèmes ${ }^{18}$." Le livre a donc été écrit, comme La Ville radieuse, dans les années 1930 ; celles-ci sont marquées par une crise économique sans précédent, qui incite les urbanistes à prendre position, à travers l'aménagement, sur la répartition du travail et des ressources.

Le volume présente le même aspect que les titres publiés par Hilberseimer dans les années 1940-1950: grand format relié sous jaquette illustrée en couleurs, autour de 200 pages, nombreuses illustrations en noir et blanc dans une mise en pages élégante, 
presque austère, argumentation sobre et efficace en plusieurs parties clairement identifiées et rigoureusement articulées. Il est structuré en cinq parties, dont les trois dernières nous intéressent; elles abordent, avec force illustrations in et hors texte, l'histoire des villes, la révolution industrielle, l'accroissement de la population, la définition d'une région et le nouveau modèle (pattern) régional; la conclusion, «Faits, pistes et sens ", reprend l'argument mené à l'échelle territoriale. Les illustrations sont soit des photographies contemporaines, essentiellement aériennes, d'établissements humains et de territoires (parfois agricoles), soit des représentations graphiquement très abouties des théories de Hilberseimer, et de leur application sur quelques sites précis, notamment l'agglomération de Chicago (fig. $\mathbf{n}^{\circ} \mathbf{6}$ ).

Figure 6

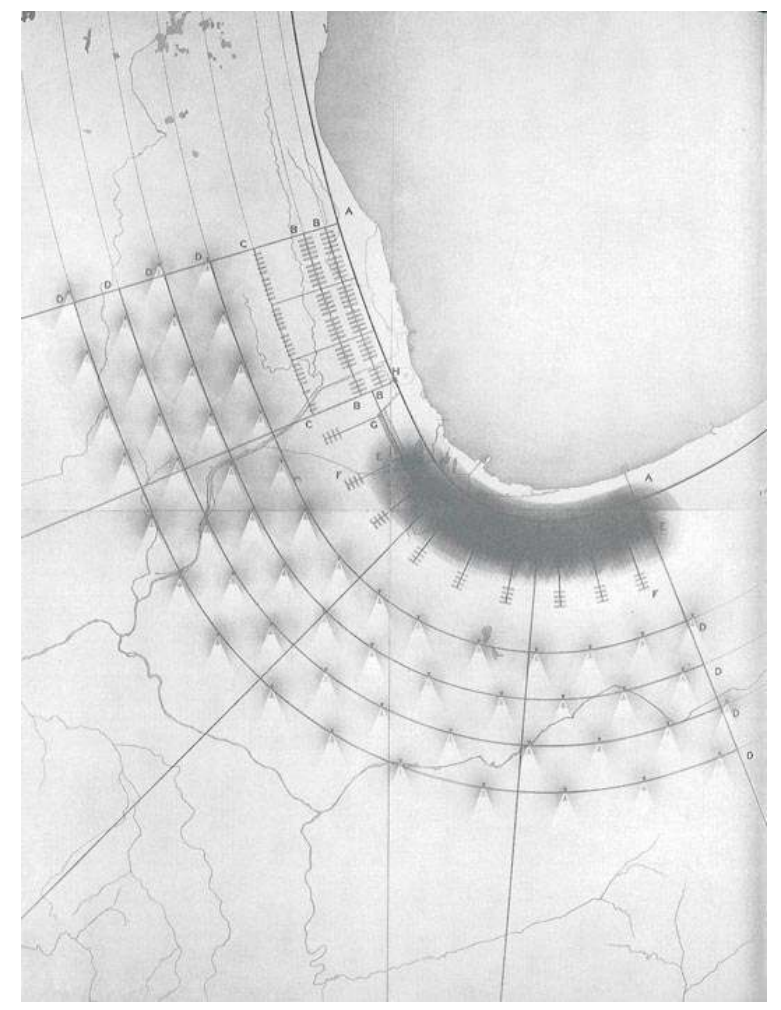

Proposition d'aménagement d'une extension pour Chicago. La représentation témoigne d'une grande virtuosité graphique. The Regional Planning, 1949, p. 148.

Repro. Morel Journel, Guillemette. (c) Guillemette Morel Journel.

On a vu que malgré l'affichage du mot «fermes » en titre, l'approche de Hilberseimer se situe à une échelle plus grande que celle de l'exploitation agricole, celle de la région. C'est avant tout de l'équilibre entre les zones urbaines et «naturelles » qu'il est question dans les hypothèses de planification. Celles-ci sont présentées dans des plans qui n'ont rien perdu de leur séduction graphique : les traits à la plume extrêmement précis, d'une rigueur toute scientifique, voisinent avec des effets de matière obtenus grâce à une grande maitrise de l'aérographe (fig. $\mathbf{n}^{\circ} 7$ ). Du point de vue théorique, les aspects humains liés au rendement du travail en temps de crise sont au cœur du propos : comment fournir aux ouvriers urbains un revenu supplémentaire, vivrier, issu de la terre? 
Figure 7

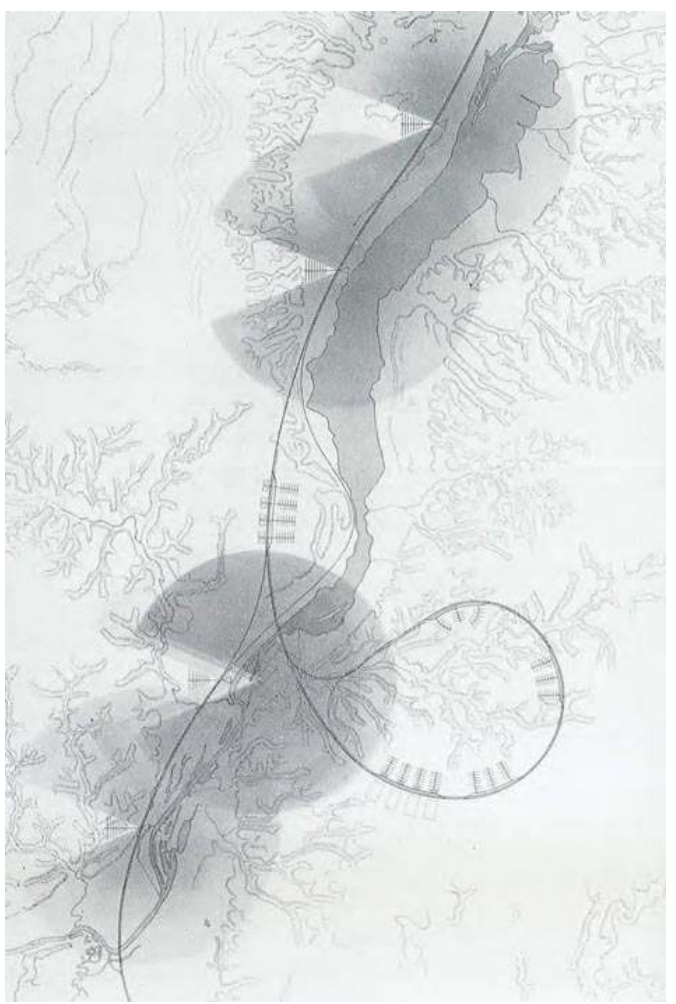

Plan de principe d'un aménagement le long d'une rivière. The Regional Planning, 1949, p. 143. Repro ?? Repro. Morel Journel, Guillemette. (c) Guillemette Morel Journel.

L'hypothèse est la suivante : pour résorber le chômage, les 8 heures journalières de travail d'un ouvrier doivent être réparties entre deux hommes - donc 4 heures chacun seulement, ce qui leur fera gagner un demi salaire : « La compensation nécessaire pour les travailleurs consisterait en des terrains pour des jardins et des fermes, selon leurs besoins. » Certes, certains ouvriers préféreront garder leur emploi à plein temps. Mais « la plupart des gens trouveront plus d'avantage et de satisfaction dans le travail mixte, entre les deux extrêmes de l'industrie pure et de la pure agriculture ${ }^{19}$ ». Cela rééquilibrerait l'économie de la production et de la consommation, renforcerait l'autonomie des salariés/paysans; l'individu comme la société dans son ensemble y trouveraient leur compte: "Dans notre schéma régional, qui combine industrie et agriculture, les établissements [humains] décentralisés se mêleraient au paysage et en deviendraient une partie. Quiconque le souhaiterait pourrait avoir un terrain à lui à cultiver et, de ce fait, l'indépendance que seule la terre peut donner. »

Cette économie du travail, solidaire avant l'heure, trouve sa justification dans le contexte de crise de conception du modèle. On trouve de nombreuses similitudes, à la fois en termes de gouvernance, de rééquilibrage ville/campagne et de partage du travail dans les propositions concomitantes, mais formellement très différentes, du paysagiste Lebericht Migge $^{20}$.

Elle est indissolublement liée à un nouveau mode d'occupation du territoire, dans lequel villes et campagnes ne sont plus rigoureusement distinctes. Cette conception dispersée de l'espace géographique est elle-même consubstantielle d'un changement d'échelle dans l'approche de l'aménagement: «La planification à grande échelle est aujourd'hui plus 
qu'une possibilité, c'est une nécessité. [...] La tendance à la décentralisation dépasse notre volonté. [...] Il nous faut un nouveau type de planification et de zoning, capable de déterminer OU et QUOI construire. [...] Avec une planification pertinente, progressive, chaque bâtiment nouveau au bon endroit, tout le schéma [pattern] urbain et rural sera changé et amélioré ${ }^{21}$.»

La prise en compte des espaces agricole et naturel à l'échelle nationale, voire planétaire, passe donc par une nouvelle vision du métier de l'urbaniste : il ne peut plus penser un aménagement quelconque sans adopter un regard global, au moins à l'échelle de la région. Cette approche compréhensive et intégrative est certainement informée par la nouvelle patrie de Hilberseimer : la tendance à la « dédensification et à la diversification » fait penser au grand modèle américain des années 1930 qu'est la Broadacre City de Frank Lloyd Wright, et à la tradition étasunienne de pensée du territoire et de la frontière. Elle est également portée par une vision utopique du bonheur très proche de celle formulée dans la Ferme radieuse: "La division-intégration du travail sera avantageuse à la fois pour les ouvriers et pour les petits fermiers. Chacun d'eux verra son standard de vie s'élever. On obtiendra un bien-être général et une économie plus équilibrée. Les conditions de vie se rapprocheront des besoins de l'homme. Il y aura du soleil dans la maison et de l'air sain partout. Plus de villes infestées de taudis. La vie redeviendra pleine de vigueur et de santé. L'indépendance et la sécurité individuelle seront renforcées, ce qui profitera à chacun et à la société à laquelle il appartient ${ }^{22}$. »

21 À rebours de la conception techniciste et centralisée de Le Corbusier, l'approche de l'émigré allemand comprend en outre une dimension écologique, qu'il développera plus encore par la suite : « La première phase de notre âge industriel a été caractérisée par une concentration et une spécialisation de la production et de l'exploitation des ressources, tant humaines que naturelles, et un intense progrès matériel. La ville et la campagne se sont dissociées. Au service de fins différentes, elles sont devenues opposées. Le second âge industriel inversera-t-il cette tendance ? Sera-t-il marqué par une décentralisation et une diversification de la production, tant industrielle qu'agricole; par l'intégration de ces deux types de production, et de celles de la ville et de la campagne? La ville va-t-elle devenir plus rurale, et la campagne plus urbaine, et toutes deux plus humaines? L'exploitation des ressources sera-t-elle remplacée par un usage planifié et une préservation attentive ${ }^{23}$ ?» Par delà leur différence de vues sur la dissémination territoriale, Hilberseimer et Le Corbusier se rejoignent dans leur adhésion à une pensée hygiéniste de l'aménagement, mais aussi la distinction qu'ils opèrent entre un premier et un second âge industriel, celui-ci corrigeant, pour le plus grand bonheur de tous, les excès de celui-là.

\section{Théorie et pratique}

Alors que le progrès technique suscite une foi presque inébranlable dans l'avenir de l'homme, les «conditions de nature » chantées par Le Corbusier semblent relever de la pure abstraction. La Terre nourricière restant chez les deux architectes un idéal désincarné, la production agricole est étudiée à grande échelle, en termes de grandes orientations régionales, et non au niveau plus concret de l'exploitation, voire des bâtiments de la ferme. La posture qu'ils adoptent, donc le statut qu'ils accordent à leurs propositions, et surtout à leurs représentations, est à cet égard révélateur. Quel qu'en soit le lien avec une réalité située, il s'agit avant tout de principes théoriques, alors même que 
leurs concepteurs sont conscients des limites de toute réflexion menée in abstracto. Écoutons-les à ce sujet.

D'abord, Le Corbusier, en 1934 : «Il ne s'agit pas ici d'une ferme particulière à un paysan donné, mais d'une étude systématique. C'est un type qui a été créé. Les divers éléments peuvent être groupés différemment selon la forme et la topographie du terrain. Mais il s'agit là d'un outil de travail pour la campagne où le progrès a apporté toutes ses ressources ${ }^{24}$.»

Pourtant l'entreprise, on l'a vu, avait commencé dès le début des années 1930 sous l'impulsion d'un « vrai » paysan, et nécessita une acclimatation du maître aux problèmes de l'espace rural : « On aborde plus facilement (nous autres!) les problèmes de l'industrie des villes que le multiple déploiement annuel du travail des champs. Toutefois, avec de la modestie et de la persévérance, les fonctions nécessaires se sont alignées dans l'ordre et dans leur forme précise, petit à petit, sur la planche à dessin. Tout s'est classé, aggloméré, ordonné, dimensionné, composé. Des plans, des coupes. [...] Et voilà : la ferme est debout. Ferme des temps modernes. Type de la ferme d'aujourd'hui. Une biologie, un organisme, un être ${ }^{25}$. "

Le statut de la Ferme radieuse est celui d'un projet à la fois théorique et concret, sans que l'on sache qui précède l'autre. Il a donné lieu à une formalisation précise à l'échelle architecturale, à la fabrication d'une maquette, à la production de nombreux dessins de production et de communication (fig. $\mathbf{n}^{\circ} \mathbf{8}$ ). La réalisation d'un prototype fut même envisagée dans le Perche, région où habitait Bézard. Le Corbusier effectua un photomontage à l'échelle du plan type d'un Village radieux sur une carte réelle du village de Bézard, qu'il légenda « Application au village de Piacé (Sarthe) ${ }^{26}$ ».

Figure 8

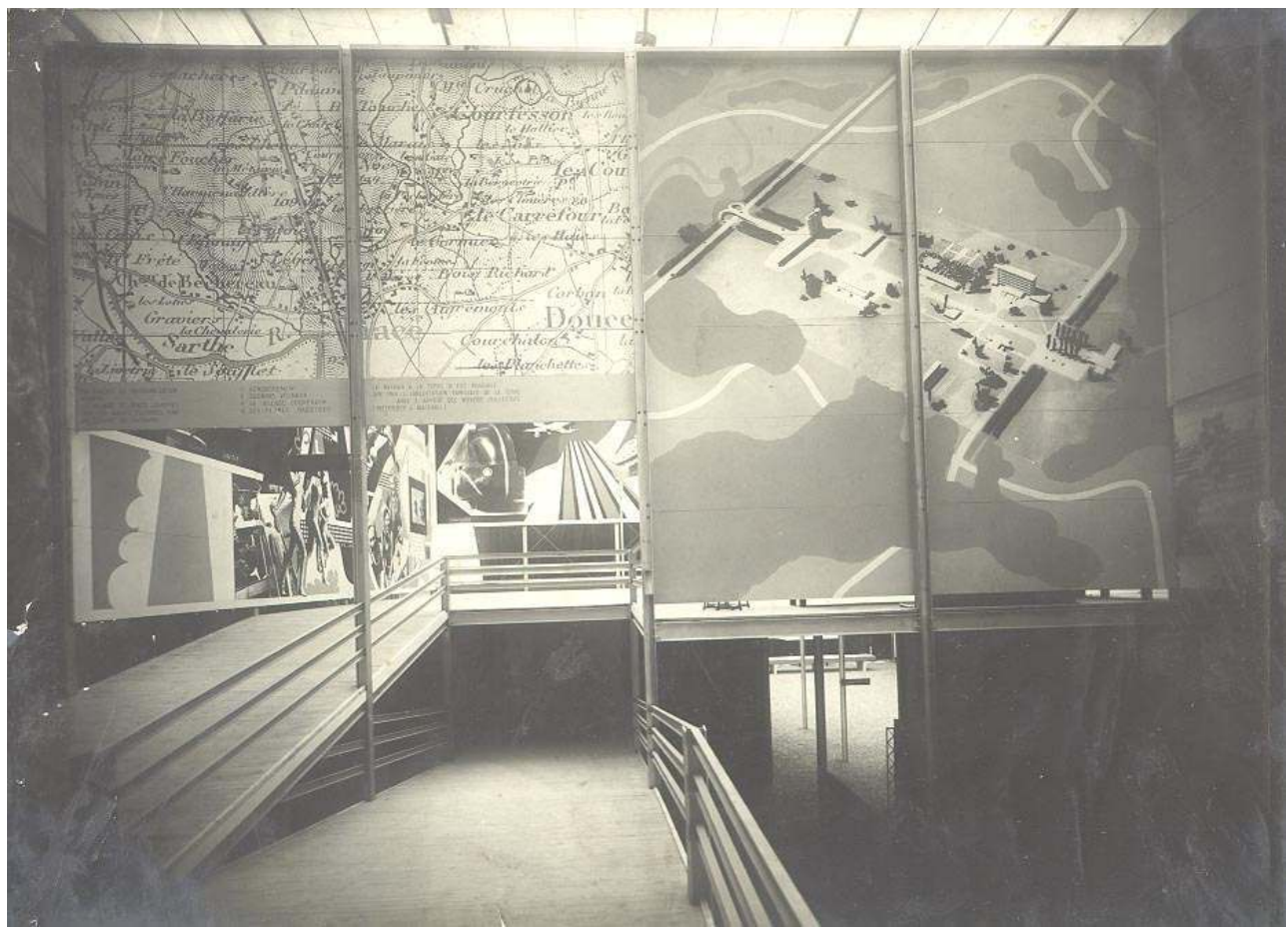

Dans le Pavillon des temps nouveaux, le site de Piacé est présenté en 1937 en regard du projet de Ferme radieuse. Fondation Le Corbusier, L2-13-95.

(c) ADAGP/FLC. 
d'interlocuteur clairement identifié et de terrain d'étude précis, Hilberseimer en fut réduit à une proposition abstraite qui ne connaîtra ni "application ", comme chez son confrère européen, ni "adaptation » - opération que Le Corbusier eût sans doute jugée trop soumise à la contingence. Il alla jusqu'à consacrer un paragraphe au «But de nos schémas » dans The New Regional Planning: «Les schémas présentés ici ne se veulent en aucun cas des solutions complètes aux problèmes soulevés. Ce sont des ébauches de solutions envisageables. Leur principal usage sera d'ouvrir le débat sur les problèmes de planification et de circulation à l'échelle locale et régionale. Les villes et les régions ne sont pas des abstractions, ce sont des entités vivantes. Comme la théorie précède les applications pratiques, les théories doivent être mises au point avant que les principales tâches de planification soient rendues possibles. [...] La planification est un travail qui englobe tout, elle nécessite une approche théorique claire. Ce n'est qu'ainsi que les vrais problèmes de l'aménagement peuvent être résolus, et que la forme du futur pourra être correctement précisée ${ }^{27}$."

Peu rompus au «travail des champs", les rares architectes du Mouvement moderne à s'intéresser aux campagnes ont adopté une vision plus théorique que pratique et produit « des ébauches de solutions envisageables » - à l'instar de celles qu'ils mirent en œuvre dans leurs projets pour la grande ville.

\section{NOTES}

1. - Rappelons que le titre du colloque dont sont issues les contributions de la présente livraison est : La ferme moderne. Architecture rurale et constructions agricoles au $X X^{e}$ siècle.

2. - Dès 1976, Manfredo Tafuri préfère parler d'« architecture contemporaine » dans le volume qu'il consacre à l'histoire $\mathrm{du} \mathrm{XX}^{\mathrm{e}}$ siècle. Sur l'évolution du concept, voir TOURNIKIOTIS, Panayotis. Historiography of Modern Architecture. Cambridge (MA) : The MIT Press, 1999.

3. - Voir ici même la contribution de Gilles Ragot, Marina Epstein-Plioulitch et Tzafir Fainholtz, "Is the kibbutz a 'radiant village' ? Le Corbusier and the Sionist movement ». Dans BALLANTYNE, Andrew (dir.). Rural and Urban, Architecture between two Cultures. New York: Routledge, 2010, p. $160-176$.

4. - La même contribution paraît, toujours en 1934, dans le $\mathrm{n}^{\circ} 4$ de la revue L'Homme réel.

5. - LE CORBUSIER. Cuvre complète 1929-34. Zurich : Girsberger, 1934, p. 186.

6. - Plus tard, ce sera au substantif de changer : " centre » sera adopté au détriment de "village ", qui ne sonnait sans doute pas assez moderne.

7. - LE CORBUSIER. Des Canons ? Des munitions? Merci... Des logements S.V.P. Boulogne-sur-Seine : Éditions de l'Architecture d'aujourd'hui, 1937.

8. - LE CORBUSIER. Sur les 4 routes. Paris : Gallimard, 1941.

9. - LE CORBUSIER. «Renaissance de la vie paysanne ». Sillons, 1939, $\mathrm{n}^{\circ} 1$. Manuscrit original : Fondation Le Corbusier (désormais FLC), boîte B3-12-181.

10. - FLC, C3-4-213.

11. - (Assemblée de Constructeurs pour une Rénovation Architecturale). Les Trois Établissements humains. Boulogne-sur-Seine : Éditions de l'Architecture d'aujourd'hui, 1945. 
12. - Respectivement Paris: Bourrelier, et Boulogne-sur-Seine: Éditions de l'Architecture d'aujourd'hui, 1946.

13. - Paris : Éditions de Minuit, 1957.

14. - Je n'ai trouvé à Paris qu'un seul exemplaires du livre, à la bibliothèque de l'École nationale supérieure d'architecture de Paris-Malaquais (cote: Rés 7479); un tampon sur la page de titre indique que ce volume est issu des collections du ministère de la Reconstruction et de l'Urbanisme (MRU, actif de 1944 à 1966).

15. - Le titre même de la seule monographie qui lui ait été consacrée à ce jour rappelle cette écrasante tutelle : POMMER, Richard (dir.). In the Shadow of Mies. Ludwig Hilberseimer, Architect, Educator, and Urban Planner. Chicago/New York: The Art Institute of Chicago/Rizzoli, 1988. Signalons également un très précieux numéro spécial de la revue italienne Rassegna, $\mathrm{n}^{\circ} 27$, septembre 1986

16. - Respectivement Stuttgart, Julius Hoffmann pour les deux premiers titres, puis Chicago, Paul Theobald.

17. - Entfaltung einer Planungsidee. Berlin, Fundamente 6, 1963.

18. - HILBERSEIMER, Ludwig. The New Regional Pattern. Industries and Gardens, Workshops and Farms. Chicago : Paul Theobald, 1949, p 197. Toutes les traductions en français sont de moi.

19. - HILBERSEIMER, Ludwig. The New Regional Pattern. Industries and Gardens, Workshops and Farms. Chicago : Paul Theobald, 1949, p. 191.

20. - Voir son ouvrage Die wachsende Siedlung nach biologischen Gesetzen. Stuttgart: Franck'sche Verlagshandlung, 1932. La contribution de Corrine Jaquand dans la présente livraison souligne combien ces propositions sont tributaires du contexte trouble de la république de Weimar.

21. - The New Regional Pattern. Industries and Gardens, Workshops and Farms. Chicago : Paul Theobald, 1949, p. 194.

22. - HILBERSEIMER, Ludwig. The Nature of Cities, Origin, Growth, and Decline. Pattern and Form. Planning Problems. Chicago : Paul Theobald, 1955, p. 258.

23. - HILBERSEIMER, Ludwig. The Nature of Cities, Origin, Growth, and Decline. Pattern and Form. Planning Problems. Chicago : Paul Theobald, 1955, p. 257.

24. - LE CORBUSIER. Euvre complète 1929-34. Zurich : Girsberger, 1934, p. 186.

25. - LE CORBUSIER. La Ville radieuse. Boulogne-sur-Seine: Éditions de l'Architecture d'aujourd'hui, p. 324.

26. - LE CORBUSIER. La Ville radieuse. Boulogne-sur-Seine: Éditions de l'Architecture d'aujourd'hui, p. 337.

27. - HILBERSEIMER, Ludwig. The New Regional Pattern. Industries and Gardens, Workshops and Farms. Chicago : Paul Theobald, 1949, p. 182.

\section{RÉSUMÉS}

Pour l'architecture du XXe siècle, les territoires ruraux semblent constituer un "impensé ». En particulier, rares sont les architectes dits «modernes " à s'être intéressés à la construction des fermes et à l'aménagement des campagnes. Le Corbusier (1887-1965) fait à cet égard figure d'exception, qui mit au point dès 1930 le concept et la formalisation de la "ferme radieuse ». Cette dernière trouva un prolongement dans les années 1940 avec la doctrine des Trois Établissements humains, qui se nourrissait également du systématisme de la Charte d'Athènes. 
Outre-Atlantique, une figure moins connue du Mouvement moderne, l'Allemand Ludwig Hilberseimer (1885-1967), émigré en 1938 à Chicago après avoir enseigné au Bauhaus de Dessau, a également réfléchi sur cette question, publiant en 1949 The New Regional Pattern. Le sous-titre de cet ouvrage richement illustré - Industries and Gardens. Workshops and Farms - indique que l'échelle rurale fait pleinement partie du propos de celui qui avait conçu, en 1924, le spectaculaire et totalement minéral projet de HochStadt. Comment des piliers du "Mouvement moderne ", focalisé sur la seule Grande Ville, en sont-ils venus à se pencher sur l'espace rural? Leur approche d'un territoire qu'ils connaissent très mal concilie-t-elle leur aspiration à la nouveauté et la prise en compte d'une population présumée réactionnaire? Leurs points de vue ont-ils eu quelque influence sur l'aménagement des campagnes, et ont-ils fait des émules?

For the $20^{\text {th }}$ century architect, rural territories seem to be a "no-go" area. In particular, so-called "modern" architects seldom show an interest in the construction of farms and the planning of the countryside. Le Corbusier (1887-1965) is an exception in this regard and from 1930 onwards he perfected the concept and the formalisation of the "radiant farm". The consequence of the latter in the 1940s was the doctrine of the Three Human Establishments, which also derived from the systematics of the Athens Charter. Both Outre-Atlantique, a much less well-known figure in the Modern Movement and Ludwig Hilberseimer from Germany (1885-1967), who emigrated to Chicago after having taught at the Dessau Bauhaus, reflected on this question, publishing The New Regional Pattern in 1949. The sub-title of this richly illustrated work - Industries and Gardens. Workshops and Farms - indicates that the rural scale formed an integral part of the proposal of he who had devised the spectacular and totally mineral project of HochStadt in 1924. How was it that such pillars of the "Modern Movement", focussed only on the City, came to lean towards rural areas? Did they reconcile their approach to a territory about which they knew very little with their yearning for novelty and their taking into account a population which they presumed to be reactionary? Did their opinions exert any influence on rural planning, and did they have any disciples?

\section{INDEX}

Mots-clés : agriculture vivrière, années 1930, dissolution ville-campagne, ferme radieuse, Ludwig Hilberseimer, Le Corbusier, Mouvement moderne, régionalisme, théories de l'aménagement du territoire

\section{AUTEUR}

\section{GUILLEMETTE MOREL JOURNEL}

Architecte, chercheuse, Observatoire de la condition suburbaine (équipe de recherche de l'École nationale supérieure d'architecture de Marne-la-Vallée), UMR AUSser, université Paris Est g.morel.journel@free.fr 